\title{
Hour Squared Times Millimole per Milliliter
}

National Cancer Institute

\section{Source}

National Cancer Institute. Hour Squared Times Millimole per Milliliter. NCI Thesaurus.

Code C85607.

Hours squared times millimoles per milliliter. 